\title{
Experimental observations of the behavior of a bubble inside a circular rigid tube
}

\author{
Shiping Wang1,2, Qianxi Wang2†, A-Man Zhang2, Eleanor Stride3
}

1 College of Shipbuilding Engineering, Harbin Engineering University, Harbin 150001, China

2 School of Mathematics, the University of Birmingham, Ring Rd N, Birmingham B15 2TS, UK

3 Institute of Biomedical Engineering, Department of Engineering Science, Old Road Campus, University of Oxford, Oxford OX3, 7DQ, UK

This paper is concerned with the dynamics of a spark-generated bubble inside a long, rigid, circular tube with two open ends submerged horizontally in a tank filled with water. The behaviour of the bubble was found to be sensitive to two geometrical parameters: the dimensionless tube radius $\alpha=R_{T} / R_{B \max }$ and the dimensionless eccentricity $\varepsilon=E / R_{B \max }$, where $R T$ is the inner radius of the tube, $E$ is the distance from the bubble centre at inception to the axis of symmetry of the tube, and $R_{B m a x}$ is the maximum equivalent bubble radius $(\sim 10 \mathrm{~mm})$. The expansion, collapse and rebound of the bubble were captured using a high-speed camera both for the case where $\alpha>1$ and $\alpha<1$, with and without eccentricity, respectively. Some new features of the bubble dynamics were observed. In particular, a bubble initiated with eccentricity in a tube for which $\alpha<1$ was seen to migrate to the distal part of the tube at the end of collapse with formation of a jet also in that direction. This is distinct from the case of a bubble collapsing near a flat surface. A similar phenomenon has been observed previously in the case of a microbubble collapsing in a blood vessel under ultrasound excitation, but was attributed to the elasticity of the vessel wall. The present study suggests that it may in fact be due simply to the geometry of the system. A cloud of microbubbles was observed shortly after the start of rebound of the bubble. Our analysis shows that the microbubbles should be generated from nuclei in tap water with radii in the range of 10-8 to 10-6 $\mathrm{m}$.

Key words: Bubble dynamics in a tube; Bubble jetting; Spark generated bubble; Cavitation generation

\section{Introduction}

Bubble dynamics have been intensively investigated theoretically, experimentally and numerically, for a wide variety of geometries. These include single bubbles in an infinite fluid domain, near a rigid surface, a free surface, an elastic surface, solid and liquid bodies as well as other bubbles (Blake and Gibson, 1987; Brennen, 1995; Brenner, 2009; Cui et al., 2018; Feng and Leal, 1997; Gong et al., 2018; Hellman et al., 2007; Klaseboer et al., 2005; Lauterborn and Kurz, 2010; Lohse, 2003; Martínez-Bazán et al., 1999a, b; Ni et al., 2012; Plesset and Prosperetti, 1977; Rayleigh, 1917; Zhang 
et al., 2015a; Zhang et al., 2015b; Zhang and Liu, 2015). The dynamics of a bubble in a tube, however, have been less thoroughly investigated in the literature.

There are nevertheless several important applications for which an understanding of confined bubble dynamics is important. Cavitation damage in hydraulic machinery and chemical processing plant have long been subjects of interest to the engineering community (Ji et al., 2015; Wang et al., 2018b). Bubbles are also increasingly being utilized in microfluidic systems where their confined dynamics are of crucial importance to device operation (Marmottant and Hilgenfeldt, 2004). Vascular plants contain fluid-filled vessels (xylem) to transport the water and minerals from the roots to their leaves, water shortage can introduce air bubbles into xylem and the growth of bubbles in xylem is a cause of mortality during drought (Cochard, 2006). In the medical field, suspensions of microbubbles (MBs) are injected into the blood stream as a contrast agent for ultrasound imaging. The high compressibility of MBs compared to the surrounding tissue enables visualization of blood vessels down to the capillary level and MBs are thus widely used in clinical diagnostic ultrasound (Dindyal and Kyriakides, 2011; Hosny et al., 2013). Despite their clinical benefits, however, the potentially damaging effects of MBs oscillating in close proximity to blood vessel walls have remained a concern (Chen et al., 2011; Miller and Quddus, 2000).

For the case of a bubble confined in a tube where the tube/bubble radius ratio is large and the bubble is far from the tube wall its behaviour can be approximated by using the so-called "image bubble" method. At the opposite extreme, if the bubble radius is large compared to the tube radius, its behaviour can be approximated using a one-dimensional (1D) model of a cylindrical bubble (Geng et al., 1999; Og̃uz and Prosperetti, 1998; Ory et al., 2000; Sassaroli and Hynynen, 2003; Sassaroli and Hynynen, 2005; Yin et al., 2004; Yin and Prosperetti, 2005a, b). The dynamics of a laser-generated vapor bubble inside a microscale tube were studied both experimentally and theoretically using the 1D model by Sun et al. (2009), who found that it was necessary to account for thermal effects in order to accurately predict bubble behavior.

The intermediate case of a bubble confined in a tube of slightly larger dimensions has also been considered by several authors. Miao et al. (2008), Martynov et al. (2009), Martynov et al. (2011) and Qin and Ferrara (2007) have all simulated axisymmetric bubble dynamics in a deformable tube subject to ultrasound using a combination of the Finite Element Method (FEM) and Boundary Element Method (BEM). Wang et al. (2018a); Wang et al. (2013) also studied the axisymmetric motion of a 
bubble in an elastic vessel using a BEM model, and observed that two counter propagating jets are formed along the axis of symmetry during collapse. Aghdam et al. (2015) investigated the dynamics of a spark-generated bubble having a radius of $O(1) \mathrm{mm}$, inside a vertical circular rigid tube with the lower end closed and the upper end open. It was not clear however whether the asymmetrical behavior observed was due purely to buoyancy or the fact that the liquid in the tube was confined at one end.

In this study, we aim to study these phenomena further for the case of a spark-generated bubble in a rigid circular tube with two open ends and whose length is sufficient to eliminate edge effects. We examine if and when counter propagating jets are formed along the axis of the tube and whether or not these lead to secondary jets directed towards the tube wall. High speed bubble jets may cause damage to blood vessels in clinical diagnostic ultrasound. A cloud of microbubbles was observed shortly after the start of rebound of the bubble and its possible mechanism is analyzed. This is an interesting phenomenon and has potential to benefit ultrasound diagnostic.

\section{Experiment}

The experimental setup is illustrated in figure 1a. Circular polymethyl methacrylate tubes with two open ends were submerged with their long axis parallel to the base of a cubic tank of side $0.5 \mathrm{~m}$ filled with untreated tap water. Tubes with three different inner radii were used $\left(R_{T}=10,17.5\right.$ or 20 $\mathrm{mm}$ ), with corresponding lengths of 160,190 or $190 \mathrm{~mm}$. The wall thickness was $5 \mathrm{~mm}$. The experiments were performed at atmospheric pressure and the ambient room temperature $\left(\sim 24{ }^{\circ} \mathrm{C}\right)$.

Bubbles were generated according to the method published by Turangan et al. (2006) and Fong et al. (2009) using a $6600 \mu \mathrm{F}$ capacitor, charged by a $200 \mathrm{~V}$ DC source. Copper electrodes extending from the capacitor poles were crossed at a pre-determined position at the middle cross-section of the tube. When the electrical circuit was closed, a sufficiently intense current passed through the point where electrodes touched, to rapidly vaporize the water in that region and form a bubble. The copper electrodes used were thin (diameter $0.11 \mathrm{~mm})$, compared to the bubble radius $(\sim 10 \mathrm{~mm})$ and thus their influence on the bubble dynamics was expected to be small. Images of the subsequent motion of the bubble were captured using a Phantom V12.1 high-speed camera operating at 20,000 frames per second (FPS), with an AF NIKKOR 80-200mm lens. Illumination was provided by a $2 \mathrm{~kW}$ spot light positioned opposite to the camera through a diffuser (matte glass). The images were taken in the direction perpendicular to the symmetry plane formed by the axis of the tube and the bubble centre at inception.

In analysing the images, the reference length was chosen as the maximum equivalent bubble 
radius $R_{B m a x}$, determined from the maximum area $A$ of the two dimensional image of the bubble: $R_{B m a x}$ $=\sqrt{A / \pi}$. Two geometrical parameters were calculated for each experiment: the dimensionless tube radius $\alpha$ and the dimensionless eccentricity $\varepsilon$ defined as follows:

$$
\alpha=\frac{R_{T}}{R_{B \max }}, \quad \varepsilon=\frac{E}{R_{B \max }},
$$

where $R_{T}$ is the inner radius of the tube, and $E$ is the distance from the bubble centre at inception to the axis of symmetry of the circular tube, as shown in figure $1 b$.

The length scales of the system have been selected both to enable the bubble dynamics to be captured by high speed imaging and to minimize the number of variables involved. Since the maximum bubble radius is $\sim 10 \mathrm{~mm}$, surface tension effects are expected to be very small (Leighton, 1994). Buoyancy is similarly negligible in the experiments, since the associated gravity parameter is also small $\delta=\sqrt{\rho g R_{\text {Bmax }} /\left(p_{a m b}-p_{V}\right)} \approx 0.03$, where $\rho$ is the density of water, $g$ is the acceleration of gravity, $p_{a m b}$ is the ambient pressure and $p_{V}$ is the saturated vapour pressure (Li et al., 2017; Li et al., 2018). The Reynolds number corresponding to the bubble collapse has $O\left(10_{2}\right)$ and thus viscous effects should also be insignificant.

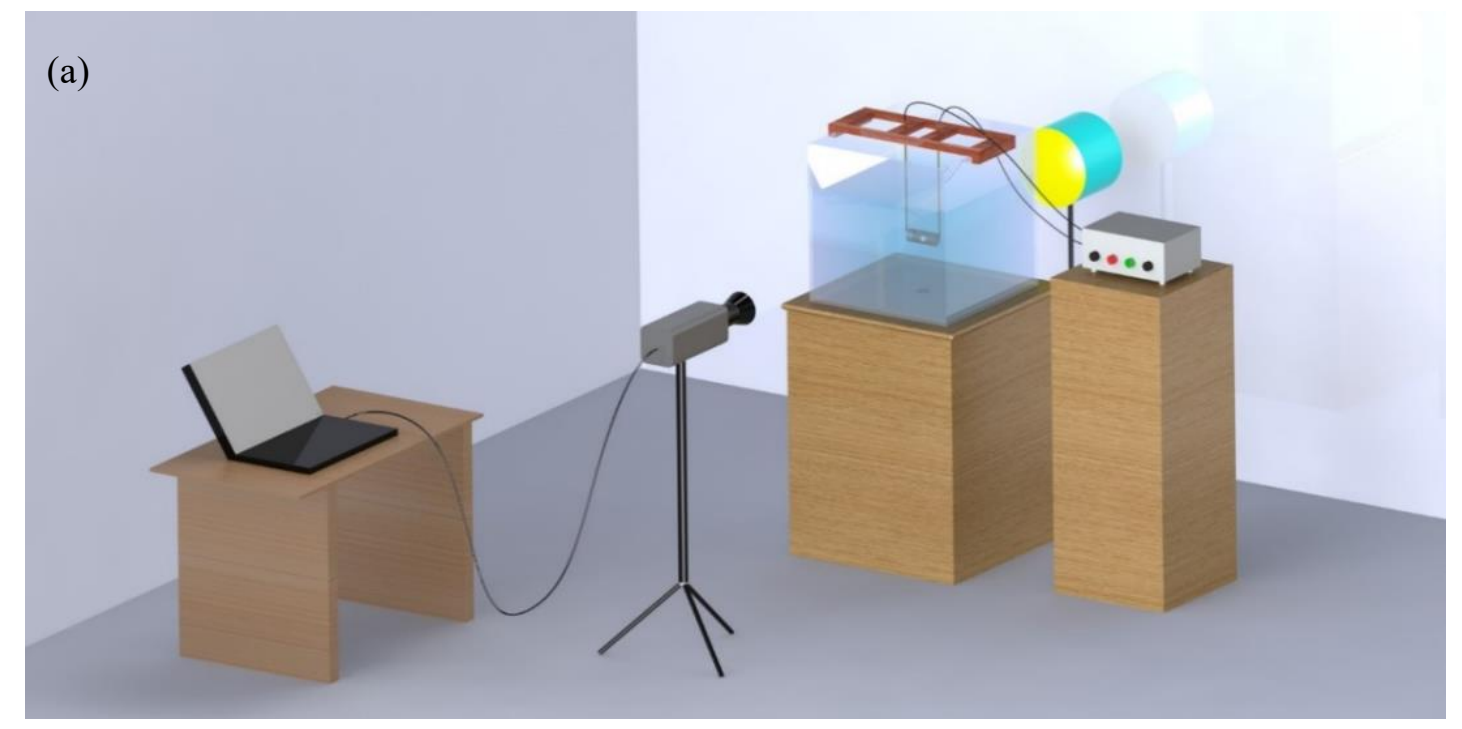




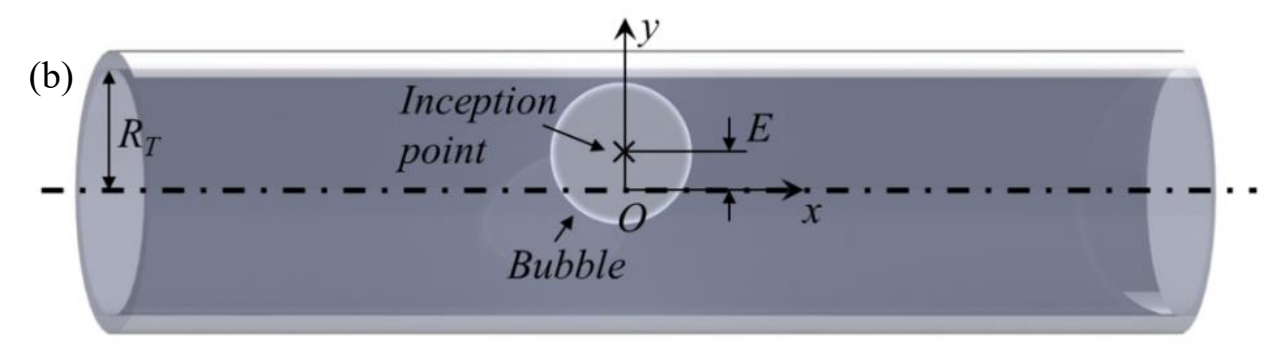

Figure 1. (a) Schematic representation of the experimental setup for a spark generated bubble in a circular tube with two open ends, which is submerged horizontally in a square water tank. (b) A bubble is initiated in a tube with the inner radius $R_{T}$ and eccentricity $E$ from the axis of the tube.

\section{Results}

\subsection{A bubble initiated on the axis of a circular tube for $\alpha>1$}

We first consider a bubble initiated on the axis of symmetry of a circular tube for the case $\alpha>1$, i.e., the tube radius is larger than the maximum equivalent bubble radius. The bubble shapes at various times for $\alpha=2.13$ are shown in figure 2. Having a higher internal pressure than the surrounding liquid at inception, the bubble initially expands pushing away liquid. The bubble becomes elongated along the axis during this phase (frame a), due to the tube wall constraining the outward liquid flow in the cross-sectional plane. The red cross ' $x$ ' in the first frame shows the initial position of the bubble. The bubble reaches its maximum volume in frame $b$. It then starts to collapse with the reduction in radius being more pronounced along the main tube axis, again due to the retarding effect of the tube wall on the liquid flow (frames $b-h$ ). The two ends of the bubble surface become flattened during the middle stage of collapse (frame c, d, e) and the bubble subsequently collapses to a thin doughnut shape at the end of collapse (frames $\mathrm{f}, \mathrm{g}, \mathrm{h}$ ). These observations can be explained by the formation of two inward jets forming along the axis of symmetry during the collapse. These counter propagating jets penetrate the bubble and subsequently collide with each other. The impact of the two jets results in a ring shape jet pointing to the tube wall, as reported in (Wang et al. 2018a).

Interestingly we observed that a cloud of microbubbles was generated rapidly in the tube, shortly after the start of rebound (frame i-1). We have observed this phenomenon in all of the tests performed. The microbubbles appeared simultaneously at various distances from the bubble, indicating that they were not generated by the fragmentation of the bubble due to surface instability. We believe that this cloud is generated by the very low pressures produced by the combinational effects of the violent collapse of the bubble and constraint of the tube wall (see section 3.5). It should be noted that microbubbles or "secondary cavitation" generated by violently collapsing bubbles have been reported in other situations and attributed to the shockwaves emitted during bubble collapse (Felix and Ellis 
1971; Lauterborn 1974, figure 8). The sudden appearance of an ensemble of tiny bubbles at the base of the microjet formed near solid surfaces has similarly been attributed to the shockwave from a collapsing bubble (Lauterborn and Bolle 1975; Lechner et al. 2017, figure 4).

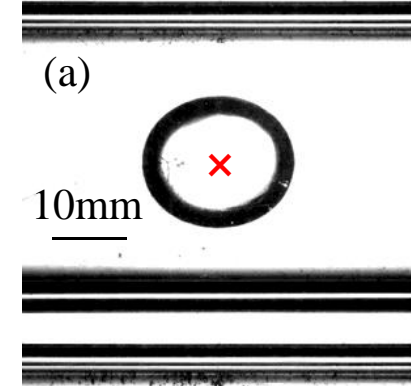

(e)
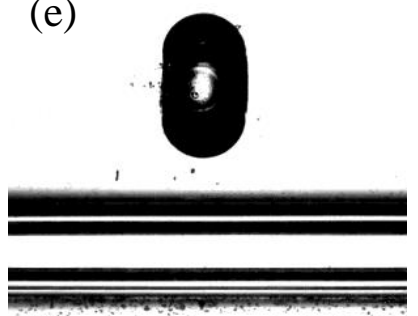

(i)

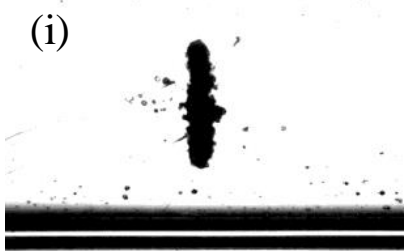

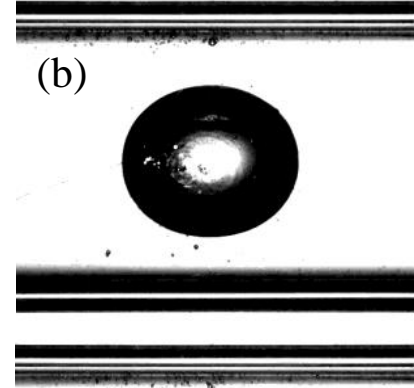

(f)
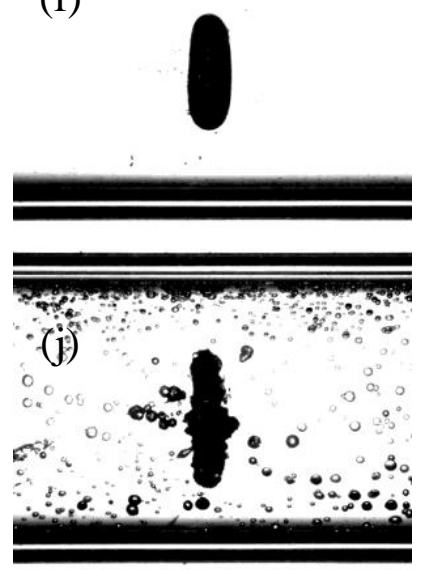

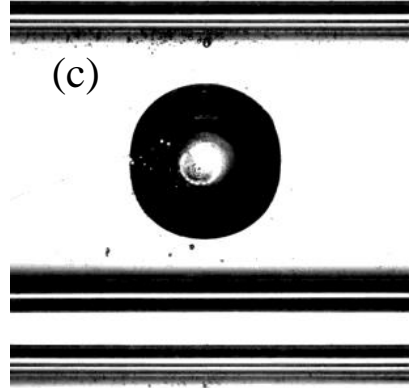

$(\mathrm{g})$
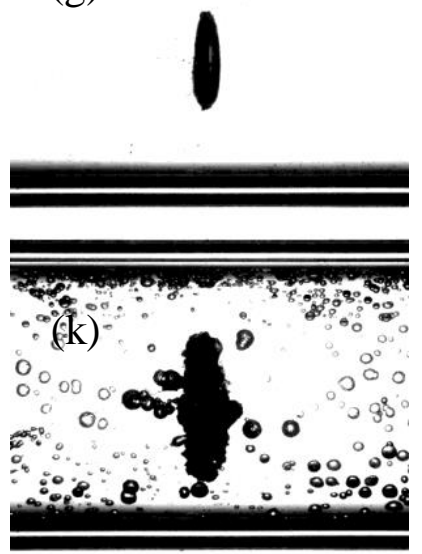

(d)

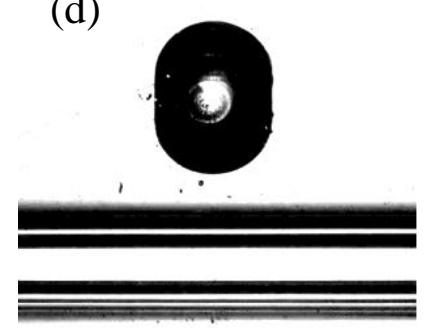

(h)
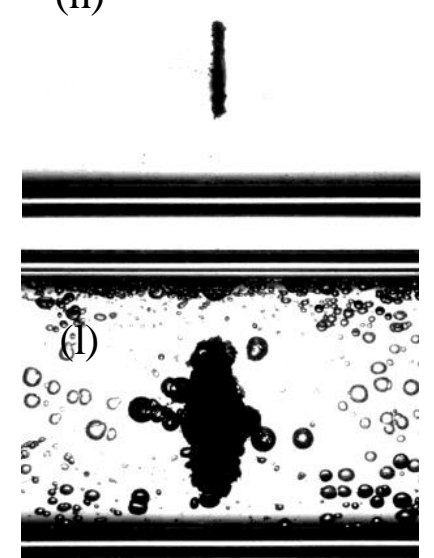

Figure 2. Bubble dynamics in a circular tube without eccentricity for the maximum equivalent radius $R_{B \max }=9.4 \mathrm{~mm}$ and dimensionless tube radius $\alpha=2.13$, at time $t=2.47,4.06,5.35,6.00,6.59,7.29$, $7.47,7.53$, and $7.941,8.12,8.35,8.71 \mathrm{~ms}$, corresponding to frames (a-f), respectively.

The next case is that of a bubble initiated on the axis of symmetry of a circular tube with a smaller dimensionless radius, $\alpha=1.64$. As may be seen in figure 3, compared to the case for $\alpha=2.13$, the bubble becomes more elongated along the axis of symmetry during expansion (frames a, b), and exhibits a more asymmetric collapse (frames b-g), due to the greater constraining effect of the tube wall. There is noticeably flattening of bubble surface in frame c, leading to two counter propagating jets being formed along the axis of the tube (frames c-e). The two jets meet at the end of collapse (frame e), generating a ring jet (frame f). Counter propagating jets were similarly observed during the collapse of strongly elongated bubbles in free liquid (Tsiglifis and Pelekasis, 2007). The bubble collapses to a thin doughnut shape at the end of collapse (frame g); meanwhile the ring jet penetrates through the middle vertical cross-section of the bubble, dividing the bubble into two parts, and then impacts on the tube wall. A microbubble cloud again develops shortly after the start of the rebound 
(frame h).
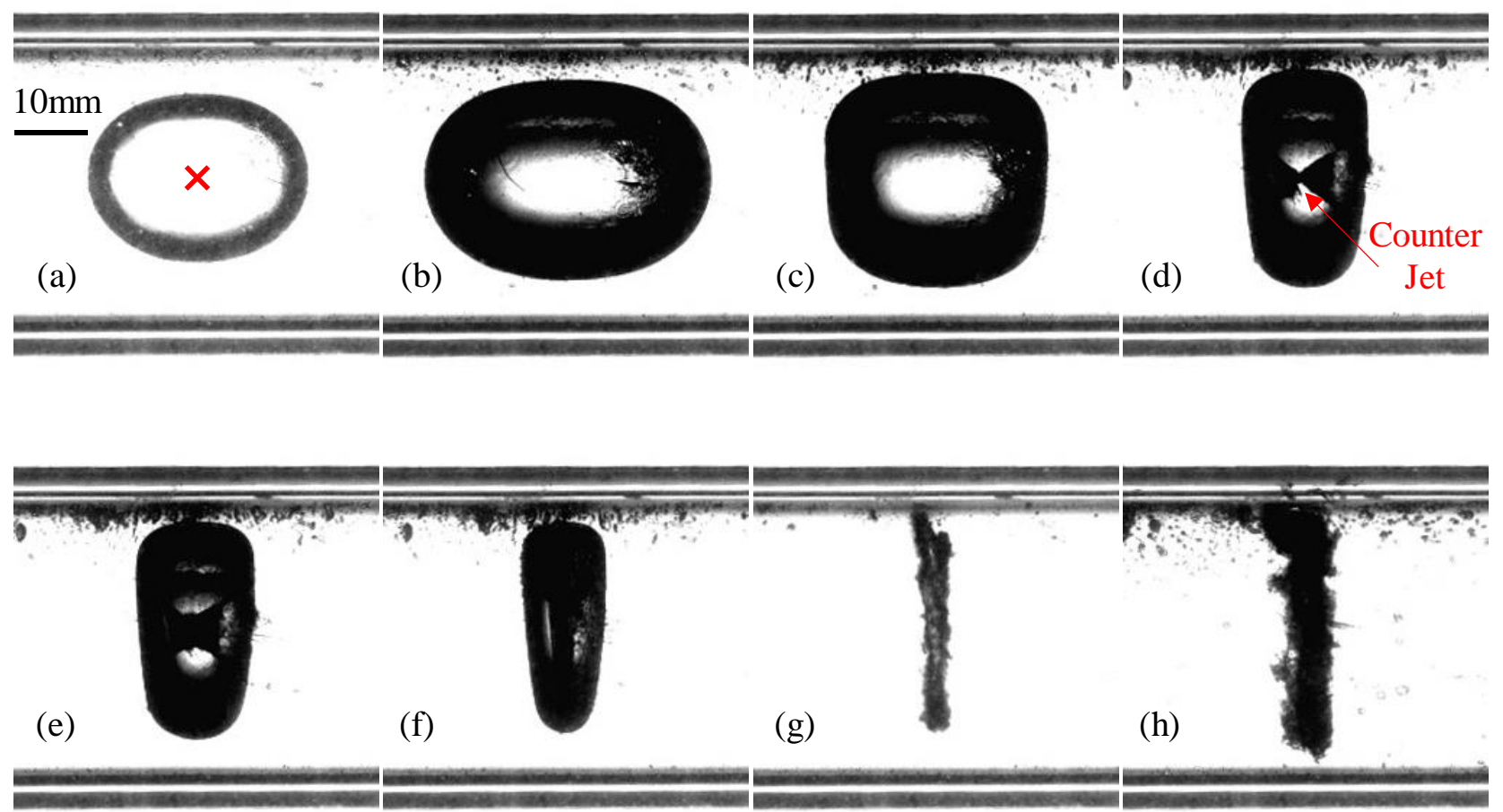

Figure 3. Bubble dynamics in a circular tube without eccentricity for $R_{B \max }=10.7 \mathrm{~mm}$ and $\alpha=1.64$, at time $t=2.48,4.62,6.76,8.71,8.86,9.47,10.05$, and $10.52 \mathrm{~ms}$, corresponding to frames (a-h), respectively.

\subsection{A bubble initiated on the axis of a circular tube for $\alpha<1$}

We next consider a bubble initiated on the axis of a thin circular tube for $\alpha<1$, i.e., the tube radius is smaller than the maximum equivalent bubble radius. The bubble shapes at different times for $\alpha=0.7$ are shown in figure 4 . The bubble adopts a long ellipsoid shape during the early stage of expansion due to the tight constraint of the tube wall (frame a). It expands mainly along the tube axis, reaching its maximum volume in frame $b$, at which point it is approximately cylindrical in shape and fully constrained by the tube wall. It then collapses from its two end surfaces (frames b-e), with two inward pointing liquid jets forming during the early stage of collapse (frame c), and impacting each other subsequently at the end of collapse (frame e). As compared to the case for $\alpha=1.64$ (figure 3), jet formation occurs earlier, their velocity is much higher (figure 5) and they meet before the bubble reaches its minimum volume (frame e). This results in an annular liquid jet that splits the bubble into two sub bubbles (frames e, $\mathrm{f}$ ). Cavitation is once again generated at the start of rebound (frame f). 

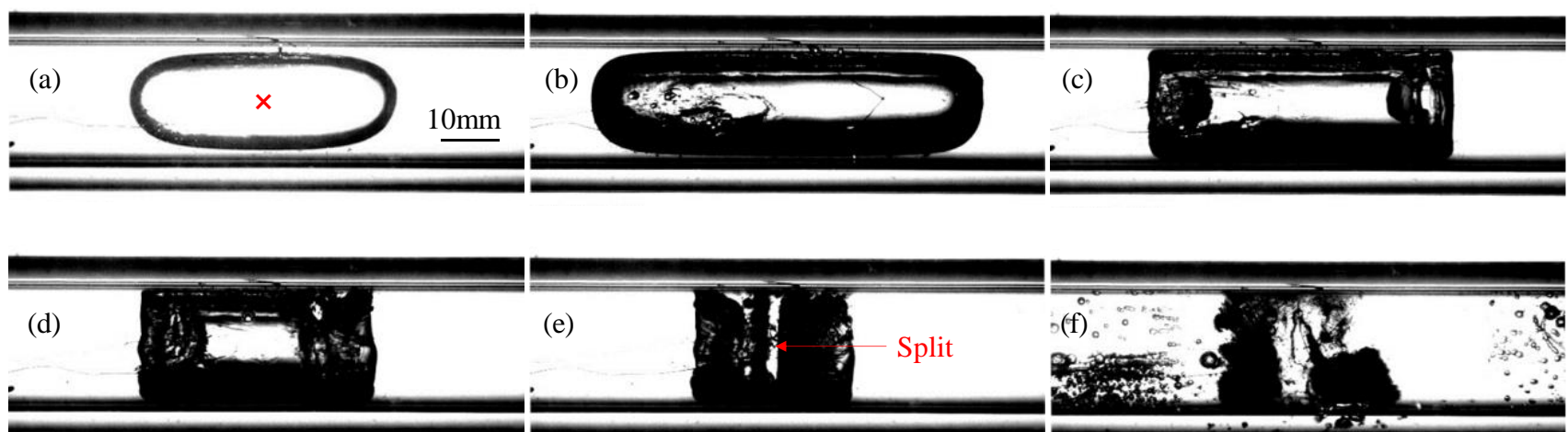

Figure 4. Bubble dynamics in a circular tube without eccentricity for $R B \max =13.6 \mathrm{~mm}$ and $\alpha=0.7$, at time $t=3.12,5.53,10.41,11.71,12.94$, and $14.00 \mathrm{~ms}$, corresponding to frames (a-g), respectively.

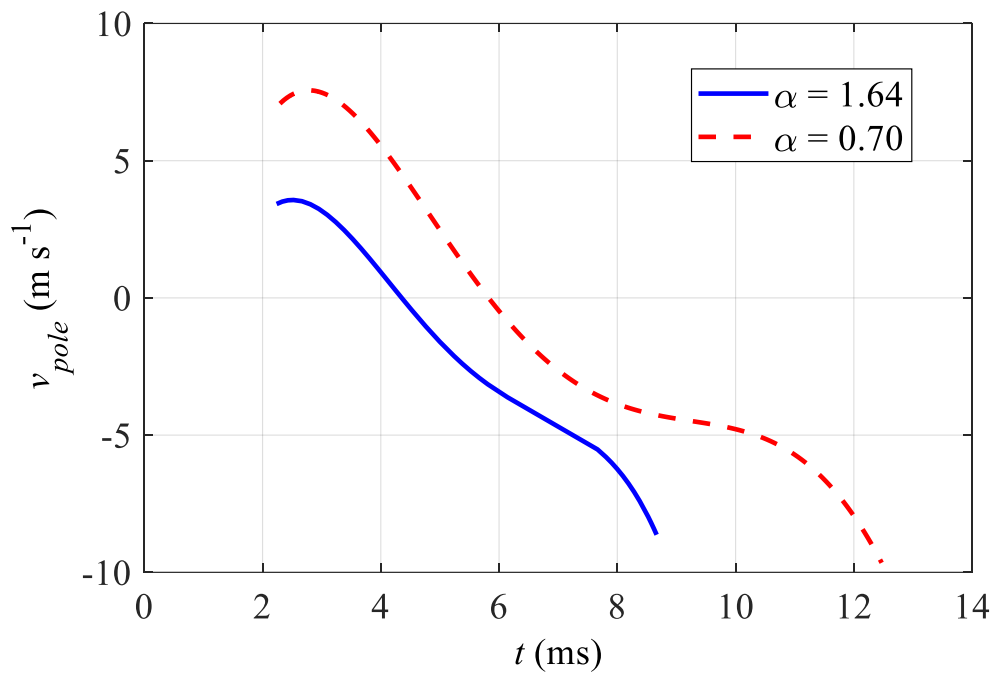

Figure 5. Time history of the velocity of the right pole $v_{\text {pole }}$ of the bubble surface for the axisymmetric cases in figures 3 and 4 for $\alpha=1.64$ and 0.7 , respectively.

Figure 5 shows how the velocity $v_{\text {pole }}$ of the right pole of the bubble surface, more specifically at the centre point of the right end of the bubble surface on the axis of symmetry, varies with time for the bubbles shown in figures 3 and 4 for $\alpha=1.64$ and 0.7 , respectively. The velocity profiles are obtained by tracking the jet front in the images. For $\alpha=1.64$, the bubble expands rapidly at inception, $v_{\text {pole }}$ reaches its maximum value $v_{\text {pole }}=3.6 \mathrm{~m} \mathrm{~s}-1$ at about $t=2.52 \mathrm{~ms}$. It then decreases with time as the bubble expands until $t=4.48 \mathrm{~ms}$, when the bubble reaches its maximum volume. After that the bubble collapses, the two end surfaces move back rapidly, and two counter liquid jets are formed subsequently. Correspondingly the jet velocity increases rapidly, reaching the maximum magnitude $v_{\text {pole }}=-8.63 \mathrm{~m}$ $\mathrm{S}-1$ at $t=8.67 \mathrm{~ms}$, just before the two liquid jets meet each other (frame e of figure 3). For $\alpha=0.7, v_{\text {pole }}$ displays similar features, but reaches a higher maximum velocity $v_{\text {pole }}=7.56 \mathrm{~m} \mathrm{~s}-1$ shortly after expansion at $t=2.82 \mathrm{~ms}$, and a higher maximum jet velocity $v_{\text {pole }}=-9.65 \mathrm{~m} \mathrm{~s}-1$ at point when the two 
jets meet at $t=12.47 \mathrm{~ms}$.

\subsection{A bubble initiated in a circular tube with eccentricity for $\alpha>1$}

We next consider bubble dynamics in a tube with $\alpha=2.47$ and large eccentricity ( $\varepsilon=1.93$ ), as shown in figure 6 . The bubble is initiated close to the upper tube wall, with the dimensionless standoff distance from the tube wall $\gamma=S / R_{B \max }=0.54$, where $S=R T-E$ is the dimensionless distance of the bubble centre at inception from the tube wall. This bubble dynamics in this case display some similar features to those observed near a rigid flat wall during expansion (Fig. 2h in Philipp and Lauterborn (1998)): the upper bubble surface becomes flattened by the tube wall during expansion (frame a), taking a half spherical shape at its maximum volume (frame b). It then collapses mainly parallel to the tube axis (frames b-d), forming a sharper distal part of the bubble surface away from the tube wall (frame d), which subsequently collapses to the upper tube wall (frames c-e). This is different to a bubble collapsing near a flat wall, where a jet often forms on the distal side of the bubble and impacts to the wall at the end of collapse phase (Lindau and Lauterborn, 2003; Philipp and Lauterborn, 1998; Yang et al., 2013).

Figures 7 and 8 show the cases for $\alpha=1.72, \varepsilon=0.74$ and $\alpha=1.40, \varepsilon=0.78$, respectively. Very clear counter jets can be observed in figures $7 \mathrm{~d}$-e. The counter jets collide and a ring shape bubble is formed in figure $7 \mathrm{f}$ that splits into two parts during the final stage of collapse in figure $7 \mathrm{~h}-1$. Comparing the three cases shown in figures 6-8, the following features are observed. As the dimensionless radius of the tube decreases, the bubble is more elongated along the axis during expansion, and the distal part of the bubble surface becomes sharper during the late stage of collapse. In all cases microbubbles are formed shortly after the start of rebound, and it can be seen by comparing figures $6 \mathrm{f}, 7 \mathrm{j}$ and $8 \mathrm{f}$ that their number increases as the dimensionless radius of the tube decreases. 

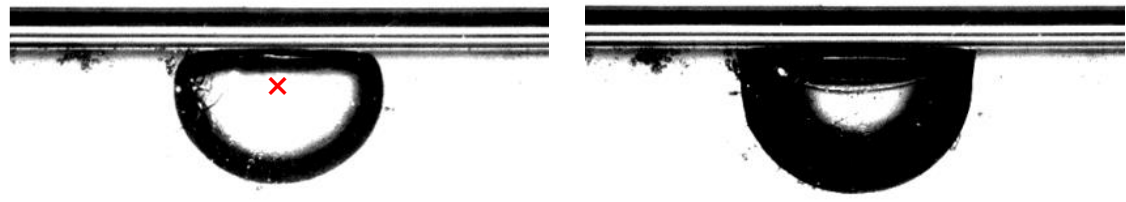

$10 \mathrm{~mm}$

(a)

(b)
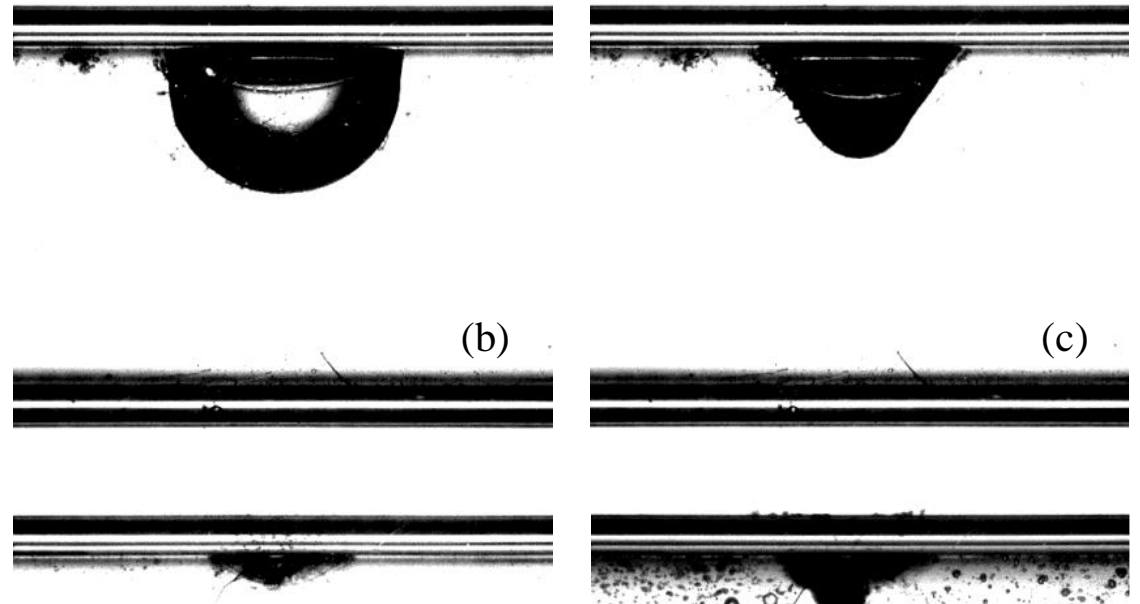

(d)

(e)

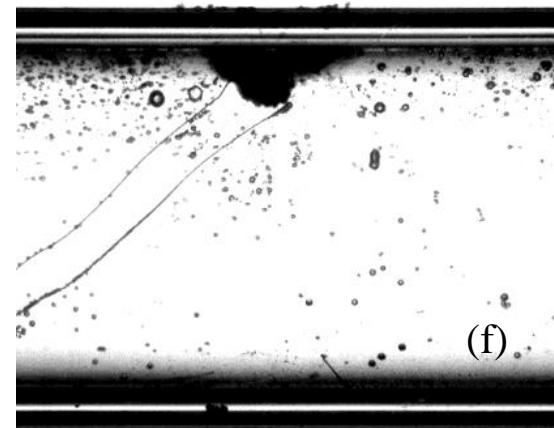

Figure 6. Bubble dynamics in a circular tube for $R_{B \max }=8.1 \mathrm{~mm}, \alpha=2.47$ and the dimensionless eccentricity $\varepsilon=1.93$ (the dimensionless standoff distance of the bubble from the tube wall $\gamma=0.54$ ), at time $t=2.24,3.59,5.35,5.82,6.29$, and $6.77 \mathrm{~ms}$, corresponding to frames (a-f), respectively.

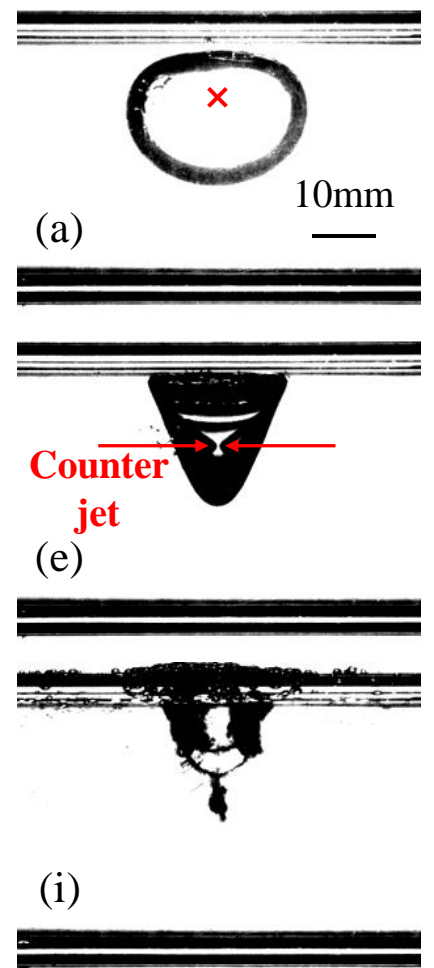

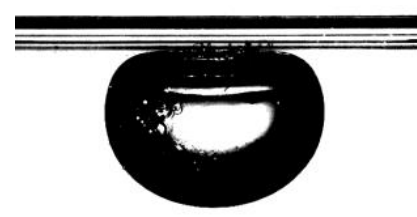

(b)

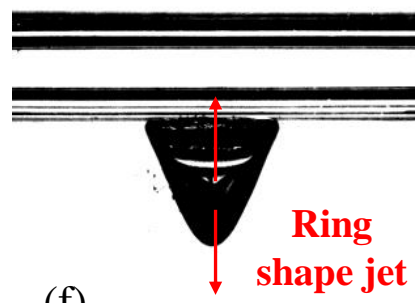

(f)

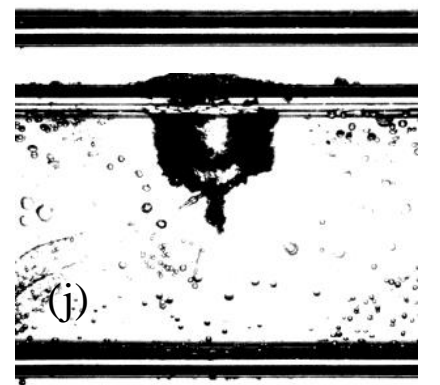

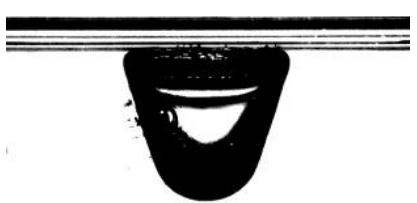

(c)

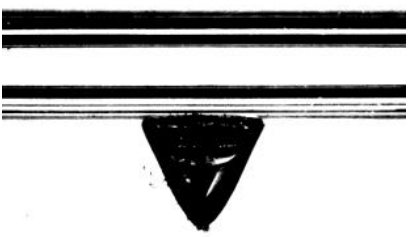

(g)

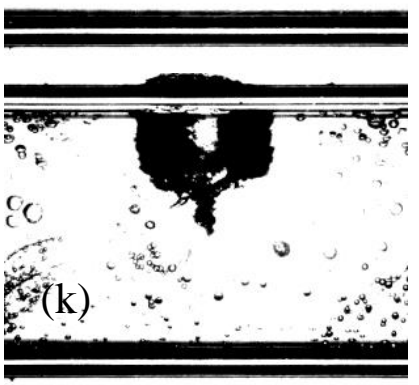

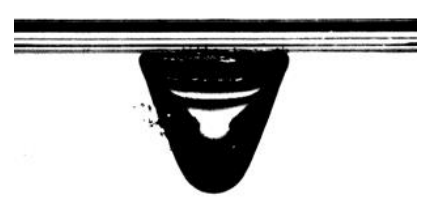

(d)

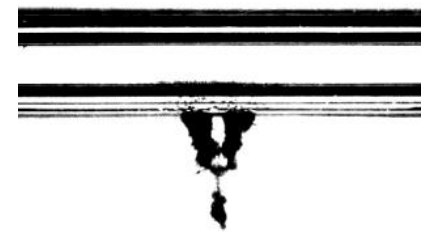

(h)

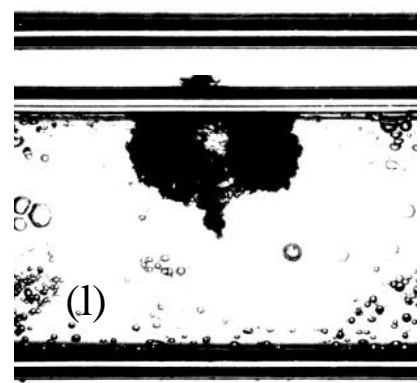

Figure 7. Bubble dynamics in a circular tube for $R_{B \max }=11.6 \mathrm{~mm}, \alpha=1.72$ and $\varepsilon=0.74$ (the 
dimensionless standoff distance of the bubble from the tube wall $\gamma=0.98$ ), at time $t=2.53,5.00$, $7.41,8.00,8.35,8.47,8.77,9.35,9.71,9.88,10.00$ and $10.29 \mathrm{~ms}$, corresponding to frames (a-1), respectively.
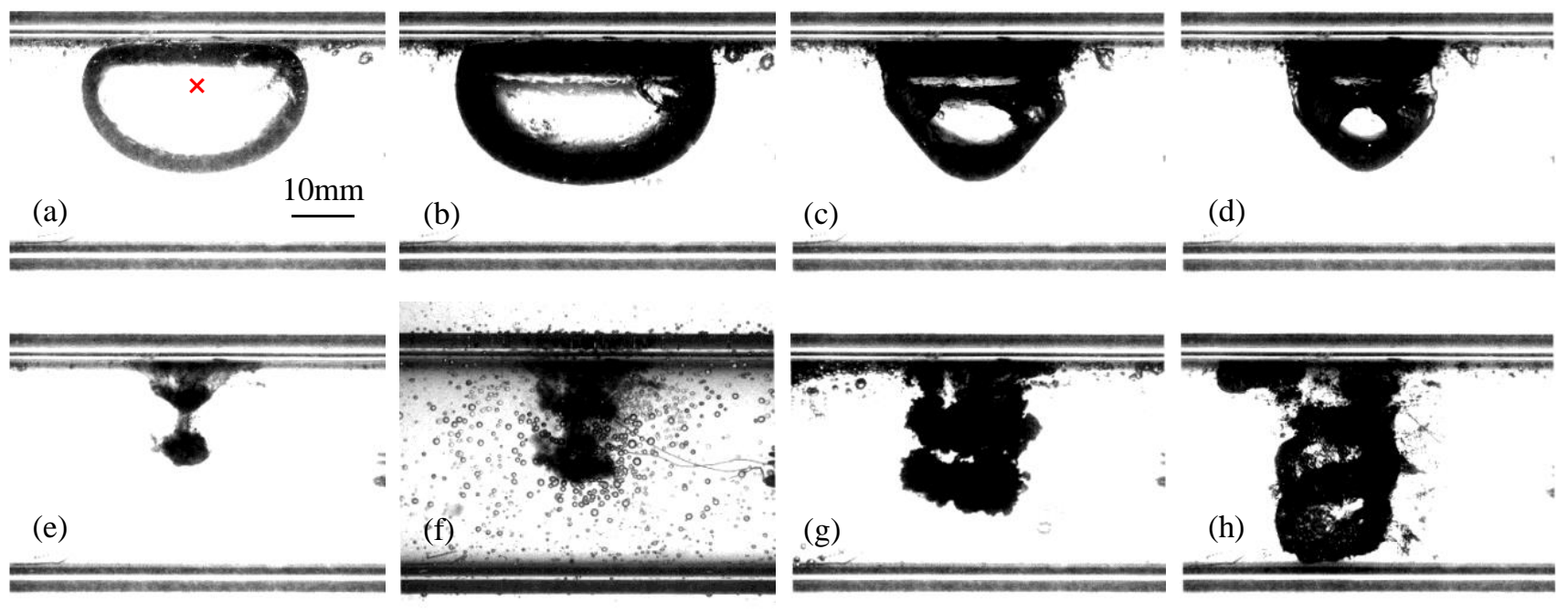

(d)

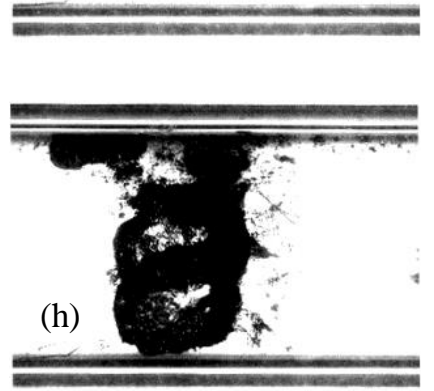

Figure 8. Bubble dynamics in a circular tube for $R_{B \max }=12.5 \mathrm{~mm}, \alpha=1.40$ and $\varepsilon=0.78$ (the dimensionless standoff distance of the bubble from the tube wall $\gamma=0.62$ ), at time $t=3.67,5.628$, $9.76,10.57,11.62,11.81,12.28$, and $16.66 \mathrm{~ms}$, corresponding to frames (a-h), respectively.

\subsection{A bubble initiated in a circular tube with eccentricity for $\alpha<1$}

Figure 9 shows a bubble in a tube with $\alpha=0.7$ and $\varepsilon=0.44$. During the early stage of expansion (frames a-b), the bubble is in contact with the upper tube wall and there is a thin layer of liquid between it and the lower tube wall. This results in asymmetry between the upper and lower parts of the bubble as can be clearly seen in frame $b$.

When the bubble starts collapsing, the upper parts of the two end surfaces contract faster than elsewhere (frames c-f). This is expected, because those parts of the bubble surface are associated with smaller radii of curvature and therefore should collapse faster, according to the proportional relationship between radius and Rayleigh collapse time (Philipp and Lauterborn, 1998). This results in two opposing liquid jets, which push away the bubble from the upper tube wall (frames d-h). The two jets impact each other (frame h), which results in a jet pointing downwards (frame i), due to the constraint of the tube wall. The downward jet subsequently breaks the bubble into two parts (frames $\mathrm{j}$, $\mathrm{k}$ ). The bubble rebounds (frames $\mathrm{j}-1$ ), reaching its second maximum volume in frame (1). Microbubble formation is observed shortly after the start of rebound (frame k). The sketches of the corresponding bubble motion on the symmetry plane formed by the axis of the tube and the center of the bubble are 
shown in figure 10 . The motion of the bubble is symmetrical with respect to this plane.
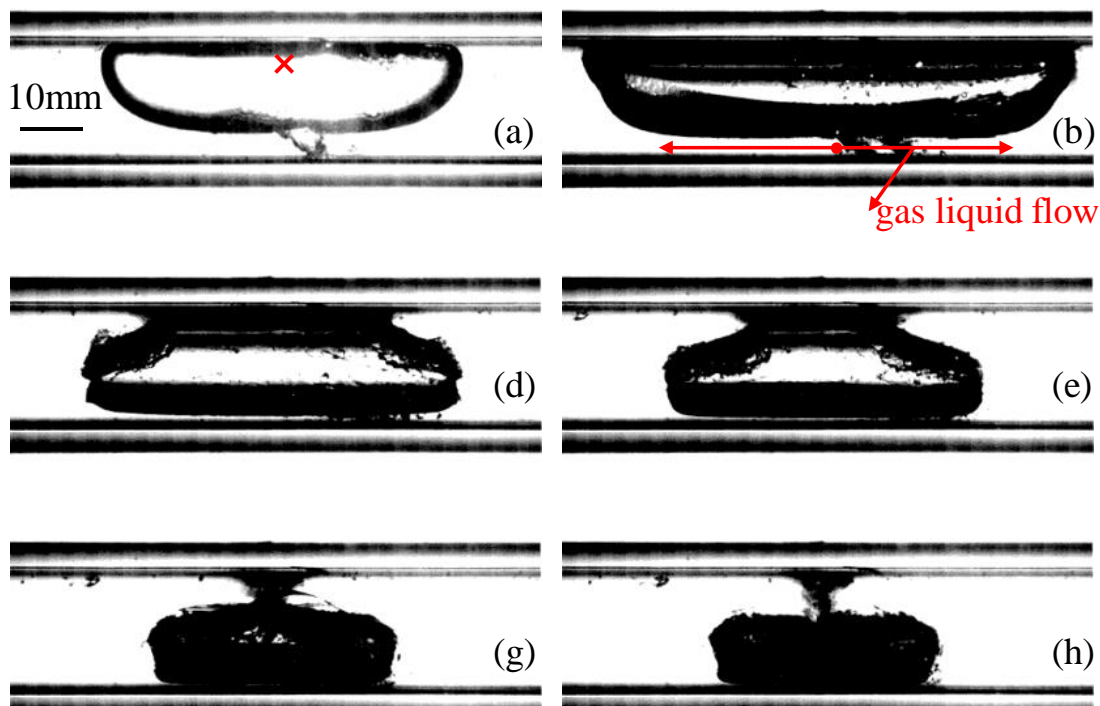

(h)

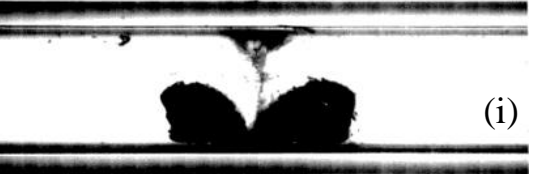

j)

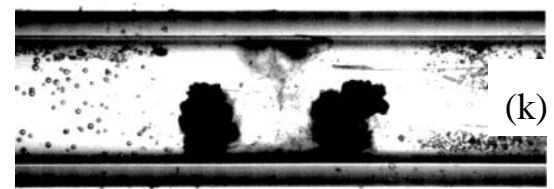

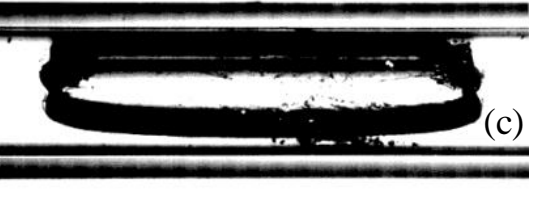

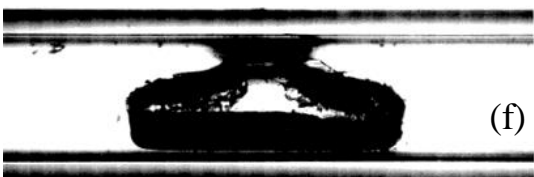

(f)
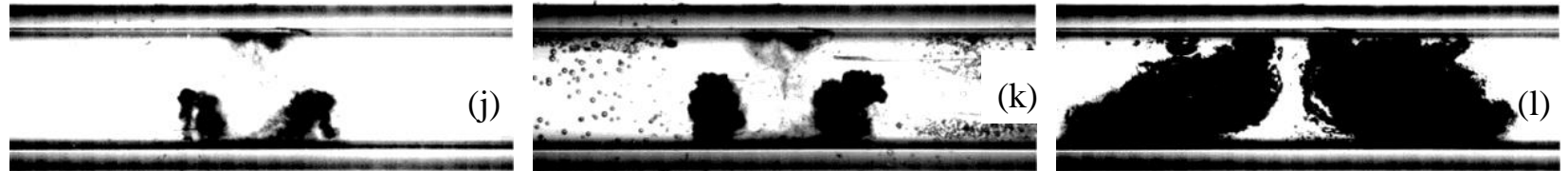

Figure 9. Bubble dynamics in a circular tube for $R_{B \max }=14.2 \mathrm{~mm}, \alpha=0.70, \quad \varepsilon=0.44$, at time $t=$ $3.91,6.63,9.31,10.89,11.98,12.77,13.36,13.71,14.26,14.65,14.95$, and $18.41 \mathrm{~ms}$, corresponding to frames (a-1), respectively.

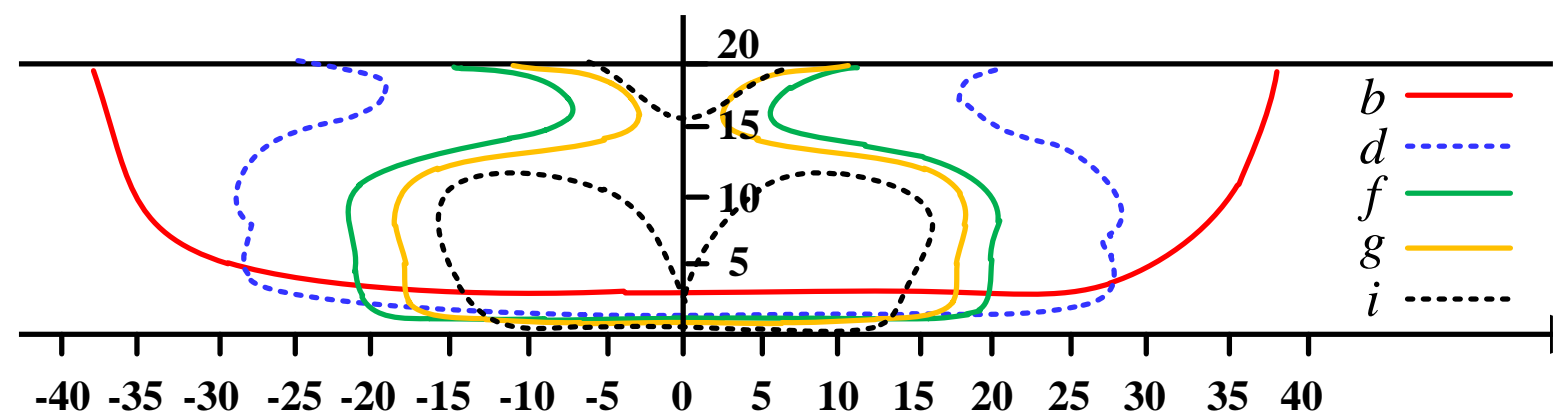

Figure 10. Sketches of bubble motion in the plane formed by the axis of the tube and the center of the bubble corresponding to frames (b), (d), (f), (g) and (i) of figure 9. 


\subsection{Analyses of microbubbles generated due to bubbles collapsing in tube}

We now discuss the possible mechanisms for the generation of microbubbles during the beginning of rebounding of spark-generated bubbles in a tube.

Free-floating nuclei are found in distilled water ranging in radii from 10-9 to 10-6 $\mathrm{m}$ and the number density decreases exponentially with increasing their radii (Leighton, 1994). Nuclei being gasfilled are evidenced by their change in size in response to pressure fluctuations in water. The dynamic boundary condition at the surface of a bubble nucleus with radius $r$ ignoring the viscous effect is given by,

$$
p_{L}=p_{s a t}+p_{g}-\frac{2 \sigma}{r}
$$

where $p_{L}$ is the liquid pressure at the nucleus surface, $p_{s a t}$ and $p_{g}$ are the saturated vapour pressure and the partial pressure of gases inside a nucleus respectively, $r$ is the nucleus radius, and $\sigma$ is surface tension.

We assume the gas pressure follows the following equation of state $p_{g}=p_{g 0}\left(r_{0} / r\right)^{3 \gamma}$, where $\gamma$ the polytropic index of gases (Gonzalez-Avila et al., 2011). Here we use the subscript "0" to denote the initial state. Substitution into (2) yields

$$
p_{L}=p_{s a t}+\frac{p_{g 0} r_{0}^{3 \gamma}}{r^{3 \gamma}}-\frac{2 \sigma}{r}
$$

$p_{L}$ first decreases and then increases with $R$, reaching its minimum value at $R=r_{c r i t .} r_{c r i t}$ and the corresponding critical liquid pressure are given as follows (Harkin et al., 1999)

$$
r_{\text {crit }}=\left(\frac{3 \gamma p_{g 0} r_{0}^{3 \gamma}}{2 \sigma}\right)^{1 /(3 \gamma-1)}, \quad p_{\text {Lcrit }}=p_{\text {sat }}+\frac{p_{g 0} r_{0}^{3 \gamma}}{r_{\text {crit }}^{3 \gamma}}-\frac{2 \sigma}{r_{\text {crit }}} .
$$

A nucleus is thus stable as $p_{L}>p_{L c r i t}$ and unstable as $p_{L}<p_{L c r i t}$, respectively.

The effect of heat transfer is measured by the Peclet number defined as $r_{0}^{2} /(\kappa T)$, where $\kappa$ is the thermal diffusivity of the gases and $T$ the time scale. The Peclet number is small for the phenomenon, considering $\alpha$ of gases are $O(10-5) \mathrm{m}^{2} \mathrm{~s}-1$, the generation of the microbubbles happens within a period of $O(10-4) \mathrm{s}$, and the radii of nuclei are in the range of 10-9 to 10-6 $\mathrm{m}$. The growth of a 
nucleus is regarded as isothermal due to the rapidity of thermal conduction over such small length scales. We therefore let $\gamma=1$. For isothermal nuclei, (4) becomes

$$
r_{c r i t}=\frac{4 \sigma}{3\left(p_{\text {sat }}-p_{\text {Lcrit }}\right)}
$$

Figure 11 shows the critical pressure in water, $p_{\text {Lcrit, }}$ versus the critical radius, $r_{c r i t}$, as governed by (5), for $p_{L 0}=1 \mathrm{~atm}, p_{\text {sat }}=0.23 \mathrm{~atm}$, and $\sigma=0.07 \mathrm{n} \cdot \mathrm{m}-1$. The magnitude of the critical pressure $p_{L c r i t}$ decreases exponentially with the critical radius $r_{\text {crit }}$ in the range of 10-9 to 10-6 m. pLcrit takes the values of $-255,-75,-7.0,-0.46,-0.01$ atm as $r_{c r i t}$ is equal to $10-9,10-8,10-7,10-6,10-5 \mathrm{~m}$, respectively. If the lowest pressure generated in the tube due to the collapse of spark generated bubbles is below $-75,-7$ atm, all nuclei in water with radii of the order of 10-8, 10-7 m, respectively, should grow to observable microbubbles. However, the exact lowest pressure in the tube generated by collapsing bubbles needs to be measured t o confirm the above analyses.

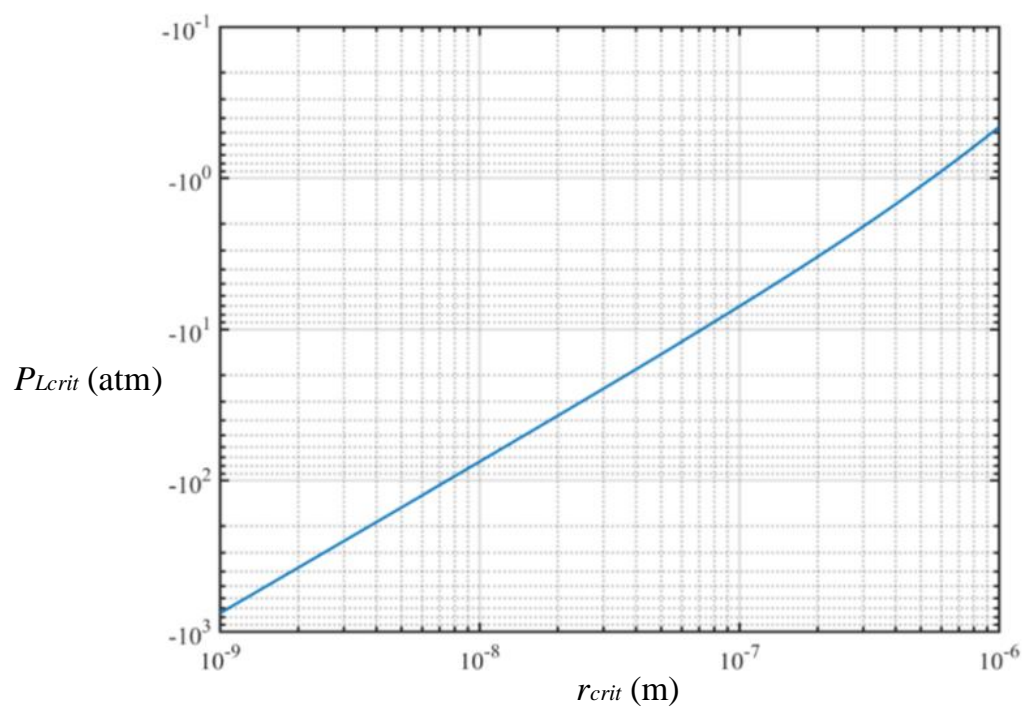

Figure 11. The critical pressure in water $p_{L c r i t}$ versus the bubble radius $r_{c r i t}$, as governed by equation (5), for $p_{\mathrm{L} 0}=1 \mathrm{~atm}, p_{\text {sat }}=0.23 \mathrm{~atm}, \sigma=0.07 \mathrm{n} \cdot \mathrm{m}-1$, and $\gamma=1$.

The generation of microbubbles cannot be attributed to evolution of dissolved gas due to heating of the liquid during bubble collapse, as the variation in liquid temperature will only be significant in the thin unsteady thermal boundary layer (Szeri et al. 2003). Similarly, fragmentation of the bubble does not provide a likely explanation. For the microbubbles to migrate from the bubble surface in frame $\mathrm{f}$ of figure 2 to $20 \mathrm{~mm}$ away in frame j, within the time period of $0.2 \mathrm{~ms}$, they would have to 
travel at a speed of $100 \mathrm{~m} / \mathrm{s}$. This is an order of magnitude larger than the maximum value of the jet velocity observed in figure 5.

The real process of cavitation is of course much more complex than the analysis above. For example, it does not take into account mass transport across the bubble surface due to condensation or evaporation. This can be modelled using Henry's law, which relates the concentration of a gas in a liquid at the bubble surface to the partial pressure of the gas inside the bubble. Similarly, the concentration of a dissolved gas in the liquid may be accounted for using the convection-diffusion equations. Fyrillas and Szeri (1994) studied dissolution or growth of soluble spherical oscillating bubbles subject to an acoustic wave. These effects could be incorporated in future development of the model although it is likely that there are other phenomena occurring on shorter timescales that also need to be taken into account for microbubble nucleation under rapid pressure changes.

\section{Implications for applications of microbubbles}

It is interesting to compare the observed phenomena in figure 9 to previous observations of jet formation reported by Chen et al. (2011). In their study, the formation of a jet directed towards the distal part of a blood vessel was observed upon the collapse of a contrast agent microbubble (Figure 12). The direction of the jet in this case was attributed to the elastic nature of the vessel wall.

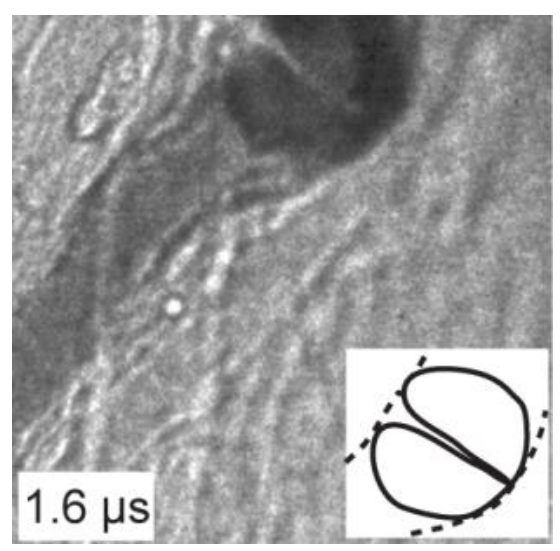

Figure 12. Single frame from a high speed video sequence capturing the collapse and microjet formation of a microbubble in rat mesentery under ultrasound excitation (Chen, 2011; Chen et al., 2011). The initial diameter of the microvessel was $15 \mu \mathrm{m}$ and the amplitude and frequency of the ultrasound pulse were $4 \mathrm{MPa}$ and $1 \mathrm{MHz}$ respectively. Inset shows a sketch of the system with the bubble and jet represented by solid lines and the microvessel wall by dashed lines. 
The results shown in figure 9 , however, suggest that it may be simply due to the geometry of the system, i.e. the tube/bubble radius ratio and initial eccentricity. There are of course some important differences between the systems considered by Chen and in the present study. In the case of a microbubble in a microscale tube, the effects of surface tension and liquid viscosity are both significant. Also, contrast agent microbubbles are primarily gas filled whereas the present study is concerned with a vapour bubble. The influence of these factors requires further investigation. 


\section{Conclusions}

In this paper, the dynamics of a bubble inside a rigid circular tube are studied experimentally using spark-generated bubbles and high-speed photography. The image sequences are analysed in terms of two geometrical parameters: the dimensionless tube radius $\alpha=R_{T} / R_{B \max }$ and the dimensionless eccentricity $\varepsilon=E / R_{B m a x}$, where $R_{T}$ is the inner radius of the tube, $E$ is the distance of the bubble centre at inception from the axis of symmetry of the tube, and RBmax is the maximum equivalent bubble radius. Some new phenomena are observed and may be summarized as follows.

If a bubble is initiated on the axis of the tube, the bubble expands and collapses primarily along this axis. During collapse two counter propagating jets are formed along the tube axis that eventually meet each other at the end of collapse. When $\alpha>2$, bubbles collapse to a thin disc shape. Whereas, when $\alpha<2$, the impact of the counter propagating jets results in an annular liquid jet, which splits the bubble into two sub-bubbles. As the tube becomes thinner, the jets form earlier and have a larger velocity.

If a bubble is initiated in a tube for which $\alpha>1$ with large eccentricity (i.e. near the tube wall), it expands asymmetrically so that the part of its surface closest to the tube wall becomes flattened. The asymmetry is accentuated during collapse with the distal part of the bubble compressing more rapidly and collapsing towards the tube wall. This is somewhat different to the case a bubble collapsing near a flat wall, which a jet often forms on the distal side of the bubble surface and impacts the wall at the end of collapse.

If a bubble is initiated in a circular tube for which $\alpha<1$ with eccentricity (saying closer to the upper part of the tube for convenience of description), its surface touches first the upper and then the lower part of the tube, forming an elongated bubble as it expands. As the bubble collapses, compression occurs mainly along the tube axis but there is more rapid movement of the bubble surface closer to the upper part of the tube. This results in two counter propagating liquid jets, that push the bubble away from the upper part of the tube and whose subsequent impact leads to a secondary jet directed towards the lower part of the tube.

In all cases studied a cloud of microbubbles was observed shortly after the start of rebound of the bubble. We hypothesize that this is due to the very low pressure produced by the violent collapse of the bubble and confinement by the tube wall. Our analysis shows that the microbubbles should be generated from nuclei in tap water with radii in the range of 10-8 to 10-6 $\mathrm{m}$. 


\section{Acknowledgements}

This work is supported by the National Natural Science Foundation of China (No. 11672082, No. 11672081).

\section{References}

Aghdam, A.H., Khoo, B.C., Farhangmehr, V., Shervani-Tabar, M.T., 2015. Experimental study on the dynamics of an oscillating bubble in a vertical rigid tube. Exp Therm Fluid Sci 60, 299-307.

Blake, J.R., Gibson, D.C., 1987. Cavitation bubbles near boundaries. Annu Rev Fluid Mech 19, 99-123.

Brennen, C.E., 1995. Cavitation and bubble dynamics. Oxford University Press, New York and Oxford.

Brenner, M.P., 2009. Cavitation in linear bubbles. J Fluid Mech 632, 1-4.

Chen, H., 2011. Ultra-high Speed Optical Imaging of Ultrasound-activated Microbubbles in Mesenteric Microvessels. University of Washington.

Chen, H., Kreider, W., Brayman, A.A., Bailey, M.R., Matula, T.J., 2011. Blood vessel deformations on microsecond time scales by ultrasonic cavitation. Phys Rev Lett 106, 034301.

Cochard, H., 2006. Cavitation in trees. Comptes Rendus Physique 7, 1018-1026.

Cui, P., Zhang, A.M., Wang, S., Khoo, B.C., 2018. Ice breaking by a collapsing bubble. J Fluid Mech 841, 287-309.

Dindyal, S., Kyriakides, C., 2011. Ultrasound Microbubble Contrast and Current Clinical Applications. Recent Patents on Cardiovascular Drug Discovery 6, 27-41.

Felix, M.P., Ellis, A.T., 1971. Laser-Induced Liquid Breakdown-a Step-By-Step Account. Appl Phys Lett 19, 484486.

Feng, Z.C., Leal, L.G., 1997. Nonlinear Bubble Dynamics. Annu Rev Fluid Mech 29, 201-243.

Fong, S.W., Adhikari, D., Klaseboer, E., Khoo, B.C., 2009. Interactions of Multiple Spark-generated Bubbles with Phase Differences. Exp Fluids 46, 705-724.

Fyrillas, M.M., Szeri, A.J., 1994. Dissolution or growth of soluble spherical oscillating bubbles. J Fluid Mech 277, 381-407.

Geng, X., Yuan, H., Og̃uz, H.N., Prosperetti, A., 1999. The oscillation of gas bubbles in tubes: Experimental results. J Acoust Soc Am 106, 674-681.

Gong, S.W., Ohl, S.W., Klaseboer, E., Khoo, B.C., 2018. Interaction of a spark-generated bubble with a two-layered composite beam. J Fluid Struct 76, 336-348.

Gonzalez-Avila, S.R., Klaseboer, E., Khoo, B.C., Ohl, C., 2011. Cavitation bubble dynamics in a liquid gap of variable height. J Fluid Mech 682, 241-260.

Harkin, A., Nadim, A., Kuper, T.J., 1999. On acoustic cavitation of slightly subcritical bubbles. Phys Fluids 11, 274287.

Hellman, A.N., Rau, K.R., Yoon, H.H., Bae, S., Palmer, J.F., Phillips, K.S., Allbritton, N.L., Venugopalan, V., 2007. Laser-induced mixing in microfluidic channels. Anal Chem 79, 4484.

Hosny, N.A., Mohamedi, G., Rademeyer, P., Owen, J., Wu, Y., Tang, M.X., Eckersley, R.J., Stride, E., Kuimova, M.K., 2013. Mapping microbubble viscosity using fluorescence lifetime imaging of molecular rotors. Proc Natl Acad Sci U S A 110, 9225-9230.

Ji, B., Luo, X.W., Arndt, R.E.A., Peng, X., Wu, Y., 2015. Large Eddy Simulation and theoretical investigations of the transient cavitating vortical flow structure around a NACA66 hydrofoil. Int J Multiphas Flow 68, 121-134.

Klaseboer, E., Hung, K.C., Wang, C.W., Khoo, B.C., 2005. Experimental and numerical investigation of the dynamics of an underwater explosion bubble near a resilient/rigid structure. J Fluid Mech 53, 387-413. 
Lauterborn, W., 1974. Kavitation durch Laserlicht. Acustica 31, 51-78.

Lauterborn, W., Bolle, H., 1975. Experimental investigations of cavitation-bubble collapse in the neighbourhood of a solid boundary. J Fluid Mech 72, 391-399.

Lauterborn, W., Kurz, T., 2010. Physics of bubble oscillations. Rep Prog Phys 73, 106501.

Lechner, C., Koch, M., Lauterborn, W., Mettin, R., 2017. Pressure and tension waves from bubble collapse near a solid boundary: A numerical approach. The Journal of the Acoustical Society of America 142, 3649-3659.

Leighton, T.G., 1994. The acoustic bubble. Academic press.

Li, S., Zhang, A.M., Han, R., Liu, Y.Q., 2017. Experimental and numerical study on bubble-sphere interaction near a rigid wall. Phys Fluids 29, 092102.

Li, S., Zhang, A.M., Wang, S., Han, R., 2018. Transient interaction between a particle and an attached bubble with an application to cavitation in silt-laden flow. Phys Fluids 30, 082111.

Lindau, O., Lauterborn, W., 2003. Cinematographic observation of the collapse and rebound of a laser-produced cavitation bubble near a wall. J Fluid Mech 479, 327-348.

Lohse, D., 2003. Bubble Puzzles. Phys Today 56, 36-41.

Marmottant, P., Hilgenfeldt, S., 2004. A bubble-driven microfluidic transport element for bioengineering. Proc Natl Acad Sci 101, 9523-9527.

MARTÍNEZ-BAZÁN, C., MONTAÑÉS, J.L., LASHERAS, J.C., 1999a. On the breakup of an air bubble injected into a fully developed turbulent flow. Part 1. Breakup frequency. J Fluid Mech 401, 157-182.

MARTÍNEZ-BAZÁN, C., MONTAÑÉS, J.L., LASHERAS, J.C., 1999b. On the breakup of an air bubble injected into a fully developed turbulent flow. Part 2. Size PDF of the resulting daughter bubbles. J Fluid Mech 401, 183-207. Martynov, S., Kostson, E., Saffari, N., Stride, E., 2011. Forced vibrations of a bubble in a liquid-filled elastic vessel. J Acoust Soc Am 130, 2700-2708.

Martynov, S., Stride, E., Saffari, N., 2009. The natural frequencies of microbubble oscillation in elastic vessels. J Acoust Soc Am 126, 2963-2972.

Miao, H., Gracewski, S.M., Dalecki, D., 2008. Ultrasonic excitation of a bubble inside a deformable tube: Implications for ultrasonically induced hemorrhage. J Acoust Soc Am 124, 2374-2384.

Miller, D.L., Quddus, J., 2000. Diagnostic ultrasound activation of contrast agent gas bodies induces capillary rupture in mice. Proc Natl Acad Sci 97, 10179-10184.

Ni, B.Y., Zhang, A.M., Wang, Q.X., Wang, B., 2012. Experimental and numerical study on the growth and collapse of a bubble in a narrow tube. Acta Mech Sinica 28, 1248-1260.

Og̃uz, H.N., Prosperetti, A., 1998. The natural frequency of oscillation of gas bubbles in tubes. J Acoust Soc Am 103, 3301-3308.

Ory, E., Yuan, H., Prosperetti, A., Popinet, S., Zaleski, A., 2000. Growth and collapse of a vapor bubble in a narrow tube. Phys Fluids 12, 1268-1277.

Philipp, A., Lauterborn, W., 1998. Cavitation erosion by single laser-produced bubbles. J Fluid Mech 361, 75-116.

Plesset, M.S., Prosperetti, A., 1977. Bubble dynamics and cavitation. Annu Rev Fluid Mech 9, 145-185.

Qin, S.P., Ferrara, K.W., 2007. The natural frequency of nonlinear oscillation of ultrasound contrast agents in microvessels. Ultrasound Med Biol 33, 1140-1148.

Rayleigh, L., 1917. On the pressure developed in a liquid during the collapse of a spherical cavity. Philosophical Magazine 34, 94-98.

Sassaroli, E., Hynynen, K., 2003. Forced linear oscillations of microbubbles in blood capillaries. J Acoust Soc Am 114, 2320-2320.

Sassaroli, E., Hynynen, K., 2005. Resonance frequency of microbubbles in small blood vessels: a numerical study. Phys Med Biol 50, 5293. 
Sun, C., Can, E., Dijkink, R., Lohse, D., Prosperetti, A., 2009. Growth and collapse of a vapour bubble in a microtube: the role of thermal effects. J Fluid Mech 632, 5-16.

Szeri, A.J., Storey, B.D., Pearson, A., Blake, J.R., 2003. Heat and mass transfer during the violent collapse of nonspherical bubbles. Phys Fluids 15, 2576-2586.

Tsiglifis, K., Pelekasis, N.A., 2007. Nonlinear oscillations and collapse of elongated bubbles subject to weak viscous effects: Effect of internal overpressure. Phys Fluids 19, 072106.

Turangan, C.K., Ong, G.P., Klaseboer, E., Khoo, B.C., 2006. Experimental and numerical study of transient bubbleelastic membrane interaction. J Appl Phys 100, 054910.

Wang, S.P., Wang, Q.X., Leppinen, D.M., Zhang, A.M., Liu, Y.L., 2018a. Acoustic bubble dynamics in a microvessel surrounded by elastic material. Phys Fluids 30, 012104.

Wang, S.P., Zhang, A.M., Liu, Y.L., Zeng, D.R., 2013. Numerical simulation of bubble dynamics in an elastic vessel. Eur Phys J E 36, 13119-13117.

Wang, Z.Y., Huang, B., Zhang, M.D., Wang, G.Y., Zhao, X.A., 2018b. Experimental and numerical investigation of ventilated cavitating flow structures with special emphasis on vortex shedding dynamics. Int J Multiphas Flow 98, 79-95.

Yang, Y.X., Wang, Q.X., Tan, S.K., 2013. Dynamic features of a laser-induced cavitation bubble near a solid boundary. Ultrason Sonochem 20, 1098-1103.

Yin, Z., Prosperetti, A., Kim, J., 2004. Bubble growth on an impulsively powered microheater. Int J Heat Mass Tran 47, 1053-1067.

Yin, Z.Z., Prosperetti, A., 2005a. 'Blinking bubble' micropump with microfabricated heaters. J Micromech Microeng $15,1683$.

Yin, Z.Z., Prosperetti, A., 2005b. A microfluidic 'blinking bubble' pump. J Micromech Microeng 15, 643.

Zhang, A.M., Cui, P., Cui, J., Wang, Q.X., 2015a. Experimental study on bubble dynamics subject to buoyancy. J Fluid Mech 776, 137-160.

Zhang, A.M., Li, S., Cui, J., 2015b. Study on splitting of a toroidal bubble near a rigid boundary. Phys Fluids 27, 062102

Zhang, A.M., Liu, Y.L., 2015. Improved three-dimensional bubble dynamics model based on boundary element method. J Comput Phys 294, 208-223. 